\title{
Effects of D-Ala ${ }^{2}$, D-Leu-Enkephalin pre- and post-conditioning in a rabbit model of spinal cord ischemia and reperfusion injury
}

\author{
DANYUN FU, HAITONG LIU, HUA LIU and JUNYAN YAO \\ Department of Anesthesiology, Shanghai General Hospital, Shanghai 200080, P.R. China
}

Received July 10, 2018; Accepted June 20, 2019

DOI: $10.3892 / \mathrm{mmr} .2019 .10729$

\begin{abstract}
It has recently been revealed that during the aorta-clamped period, D-Ala ${ }^{2}, \mathrm{D}-\mathrm{Leu}^{5}$-Enkephalin (DADLE) infusion can protect the spinal cord against ischemia and reperfusion (I/R) injury. However, the protective effects of DADLE administration prior to ischemia or at the time of early reperfusion have not yet been investigated. Drug preor post-conditioning can serve as a more valuable clinical strategy. Therefore, the present study was designed to investigate the neuroprotective effect of DADLE infusion at different time intervals in order to determine the optimum time point for ischemic spinal cord protection. A total of $40 \mathrm{New}$ Zealand white rabbits were randomly divided into 5 groups: Sham-operated (Sham), normal saline pre-conditioning (NS), DADLE per-conditioning $\left(\mathrm{D}_{\text {per }}\right)$, DADLE pre-conditioning $\left(\mathrm{D}_{\text {pre }}\right)$ and DADLE post-conditioning $\left(\mathrm{D}_{\text {post }}\right)$. All animals were subjected to spinal cord ischemia for $30 \mathrm{~min}$ followed by $48 \mathrm{~h}$ reperfusion. Hind limb motor functions were assessed according to the Tarlov criterion when the animals regained consciousness, 6, 24 and $48 \mathrm{~h}$ after reperfusion. Histological analysis and the number of viable $\alpha$-motor neurons were also used to assess the extent of spinal cord injury. Compared with the NS group, the Tarlov scores and the number of normal neurons were significantly higher in the $\mathrm{D}_{\text {per }}$ group $(\mathrm{P}<0.05)$, which were consistent with the results of a previous study. In addition, the paraplegia rate and loss of normal motor neurons were lower in the DADLE per- and post-conditioning groups compared with the DADLE pre-conditioning; however, these were not statistically significant. DADLE $0.05 \mathrm{mg} / \mathrm{kg}$ administration at three time points all mitigated normal motor neuron injury in the anterior horn and decreased the paraplegia rates in rabbits. The therapeutic benefits appeared best in the post-conditioning group with DADLE, and worst in the pre-conditioning group.
\end{abstract}

Correspondence to: Dr Junyan Yao, Department of Anesthesiology, Shanghai General Hospital, 100 Haining Road, Hongkou, Shanghai 200080, P.R. China

E-mail: sunshineyao@163.com

Key words: D-Ala ${ }^{2}, \mathrm{D}-\mathrm{Leu}^{5}$-Enkephalin, spinal cord, ischemiareperfusion injury, pre-conditioning, post-conditioning

\section{Introduction}

Spinal cord ischemia and reperfusion (I/R) injury, including permanent paraparesis or paraplegia remains a devastating complication of thoraco-abdominal aortic surgery. As previously reported, the incidence of spinal cord ischemia is $4-16 \%$ in patients having received thoraco-abdominal aortic surgery (1). Despite numerous advances in neuroprotective strategies, such as surgical techniques, hypothermia treatment and cerebrospinal fluid drainage, aiming to decrease the negative impact of I/R injury, the efficacy of each intervention has not yet been fully determined $(2,3)$.

Novel methods, such as ischemic pre- and post-conditioning have recently been demonstrated to provide protection in several organs, including the spinal cord. Compared with conventional ischemic pre-and post-conditioning, pharmacological pre- and post-conditioning, which only requires drug administration as adjunctive treatment prior to ischemia or at the time of early reperfusion, has demonstrated improved results in preventing organ $I / R$ injuries (4-6). This is due to the fact that drug administration leads to less adverse effects compared with the mechanical stimulation of vessels $(7,8)$. If the exact times of onset time for the organ ischemia can be predicted, pharmacological pre-conditioning will be more effective in improving the durability of tissue to ischemic insult. However, this method is not currently available in numerous clinical settings. From a clinical point of view, post-conditioning, which is more amenable to unpredicted ischemia in patients with thoraco-abdominal aortic aneurysm, may offer greater advantages over pre-conditioning.

Extensive previous studies have suggested that opioid receptor pre- and post-conditioning can protect tissues against $\mathrm{I} / \mathrm{R}$ injury in the central nervous system and other organ systems (9-11). D-Ala ${ }^{2}$, D-Leu ${ }^{5}$-Enkephalin (DADLE), a selective delta opioid receptor agonist, has received increasing interest as a link between hibernation and neuroprotection (12). DADLE has been revealed experimentally to improve injury to cortical neurons caused by oxygen-glucose deprivation, and also to decrease neuronal death and intellectual disability induced by forebrain ischemia $(13,14)$.

A previous study has demonstrated that administration of $0.05 \mathrm{mg} / \mathrm{kg}$ DADLE by regional perfusion into the clamped aorta during the ischemic period, could induce neuroprotective efficacy against spinal cord $\mathrm{I} / \mathrm{R}$ in rabbits (15). Whether an improved effect may be acquired when DADLE was 
delivered prior to ischemia onset or in the early reperfusion phase remains unclear. Considering the advantages of pharmacological pre- and post-conditioning in the clinical setting, the present study further compared the neuroprotective effects of DADLE administration before, during and after ischemia, in order to determine the optimal conditioning strategy.

\section{Materials and methods}

Animals and ethics. The animal protocol was approved by the Animal Care and Use Committee of Shanghai Jiaotong University, and was in accordance with the Guide for The Care and Use of Laboratory Animals (16). Efforts were made to minimize the number of animals and their suffering.

Method of anesthesia. A total of 40 New Zealand white rabbits were supplied by Animal Research Laboratory at Shanghai General Hospital Affiliated to Shanghai Jiaotong University School of Medicine. They were housed in a room under at ambient temperature $\left(20-25^{\circ} \mathrm{C}\right)$, relative humidity $40-70 \%$ and a 12-h light/dark cycle, with free access to food and water. Animals aged 4-6 months with body weight $2.0-3.0 \mathrm{~kg}$ (20 male and 20 female) were randomly divided into 5 groups $(n=8)$ : Sham-operated group (Sham), normal saline pre-conditioning (NS), DADLE per-conditioning $\left(\mathrm{D}_{\mathrm{per}}\right)$, DADLE pre-conditioning $\left(\mathrm{D}_{\text {pre }}\right)$ and DADLE post-conditioning $\left(\mathrm{D}_{\text {post }}\right)$. DADLE (Sigma-Aldrich; Merck KGaA) was dissolved in NS and the rabbits received DADLE at a dosage of $0.05 \mathrm{mg} / \mathrm{kg}$. For the rabbits in the NS and $\mathrm{D}_{\text {per }}$ groups, NS or DADLE were infused, respectively, for $30 \mathrm{~min}$ during the entire spinal cord ischemia period. The rabbits in the $\mathrm{D}_{\text {pre }}$ group received DADLE for $30 \mathrm{~min}$ prior to aortic occlusion and were then immediately subjected to the $30 \mathrm{~min}$ ischemia. The rabbits in the $\mathrm{D}_{\text {post }}$ group were given 30 min DADLE at the immediate onset of reperfusion (Fig. 1). The rabbits in the Sham group underwent the operation but the aorta was not occluded.

General anesthesia was induced with ketamine $(20-25 \mathrm{mg} / \mathrm{kg})$ and atropine $(0.06-0.10 \mathrm{mg} / \mathrm{kg})$. A catheter (22-G) was inserted into the left ear vein for venous administration. The rabbits were ventilated mechanically with volume-controlled ventilation. The parameters of mechanical ventilation were adjusted as follows: Tidal volume, $10 \mathrm{ml} / \mathrm{kg}$; respiratory rate, 30 breaths per min; ratio of inspiratory time to expiratory time, 1:1.5; and fraction of inspired oxygen, 1.0. Core body temperature was monitored and maintained at $37^{\circ} \mathrm{C}$ with a heating lamp. The right ear central artery was cannulated with a 22-G catheter for mean arterial pressure (MAP) and heart rate (HR) monitoring and blood sampling. NS containing penicillin $40 \mathrm{U}$ was infused continuously during the operation at a rate of $10 \mathrm{ml} / \mathrm{kg} / \mathrm{h}$. Midazolam $(0.5 \mathrm{mg} / \mathrm{kg})$, fentanyl $(10 \mu \mathrm{g} / \mathrm{kg})$ and vecuronium $(0.25 \mathrm{mg} / \mathrm{kg})$ were injected intermittently to maintain anesthesia.

Animal model and drug perfusion protocol. The model of 30 min aortic occlusion in rabbits was established, as previously described $(15,17)$. Briefly, under general anesthesia, the femoral arteries of the rabbits were exposed. The infrarenal abdominal aorta was exposed via abdominal incision with the ligatures placed loosely around it. Following systemic heparinization $(1 \mathrm{mg} / \mathrm{kg})$, a polycarbonate catheter $(20-\mathrm{G})$ was inserted into the aorta via femoral artery incision with the tip reaching $1-2 \mathrm{~cm}$ below the left renal artery. The end of the catheter was connected to a transducer in order to monitor aortic pressure and infuse drugs. To achieve spinal cord ischemia, the infrarenal abdominal aorta was blocked with two artery clips (Fig. 2). The ischemic period was lasted for $30 \mathrm{~min}$ and was confirmed by the presence of $<20 \mathrm{mmHg}$ of distal abdominal aortic pressure. To regain blood supply, the artery clips were removed and reperfusion was performed for $48 \mathrm{~h}$. The rabbits in the Sham group underwent the surgical procedures but the aorta was not occluded. Finally, the catheter was withdrawn and the abdomen was closed following the femoral artery ligation. The animals were extubated when normal spontaneous breathing was restored.

Neurobehavioral evaluation. The animals were scored according to the Tarlov criterion (18) at the time of regaining consciousness, 6, 24 and $48 \mathrm{~h}$ after reperfusion, respectively. The behavioral scores were graded in a scale $0-4$, with 4 being the best score: i) 0, Paralysis with no lower-limb movement; ii) 1 , weak lower-limb movement, but unable to work against gravity; iii) 2, good lower-limb motor function against gravity, but incapable of dragging legs or hopping; iv) 3, ability to drag legs and hop, but not normally; and v) 4, normal lower-limb motor function. The rabbits received a single score. According to the Tarlov scores analysis, the rabbits that scored 0 or 1 were defined as paraplegic, while those that scored 0 or 1 or 2 or 3 were defined as neurological dysfunction (18). Behavioral scores were given in a blinded manner by a laboratory personnel and then the results compared.

Histopathological examination of $\alpha$-motor neuron. The rabbits were intubated and anesthetized $48 \mathrm{~h}$ after reperfusion. Lumbar spinal cords were exposed via incision on the back at the left lateral position. Spinal cord segments of L4-L5 were removed and fixed in $10 \%$ formalin for $48 \mathrm{~h}$ at $4^{\circ} \mathrm{C}$. The rabbits were sacrificed by intravenous injection of sodium pentobarbital $(200 \mathrm{mg} / \mathrm{kg})$. Following dehydration in graded ethanol, specimens were embedded in paraffin and sliced into 5- $\mu \mathrm{m}$ thick sections for hematoxylin and eosin staining for $\sim 3 \mathrm{~h}$ at room temperature. A single anterior horn was randomly selected from two slices of spinal segments from each specimen. The number of viable $\alpha$-motor neurons in the anterior spinal cord (three horizons gathered from the vertex of the anterior horn to the central canal perpendicular) was counted under a light microscope using a $\mathrm{x} 40$ objective by an investigator blinded to the group assignment. Viable $\alpha$-motor neurons were counted based on the following standard: i) Polygonal perikarya; ii) basophilic stippled cytoplasm (containing normal Nissl bodies); and iii) round nucleus located centrally with loosely textured chromatin and prominent nucleoli (19).

Statistical analysis. Hemodynamic data (MAP/HR), body weight and core temperature are expressed as the mean \pm standard deviation. The overall difference was compared using one-way analysis of variance and repeated measures analysis of variance followed by Dunnett's test. The number of viable $\alpha$-motor neurons and Tarlov scores were compared using the Kruskal-Wallis nonparametric rank sum test followed by the Mann-Whitney U test. To obtain a $95 \%$ confidence interval value, a Bonferroni correction was used to adjust the 


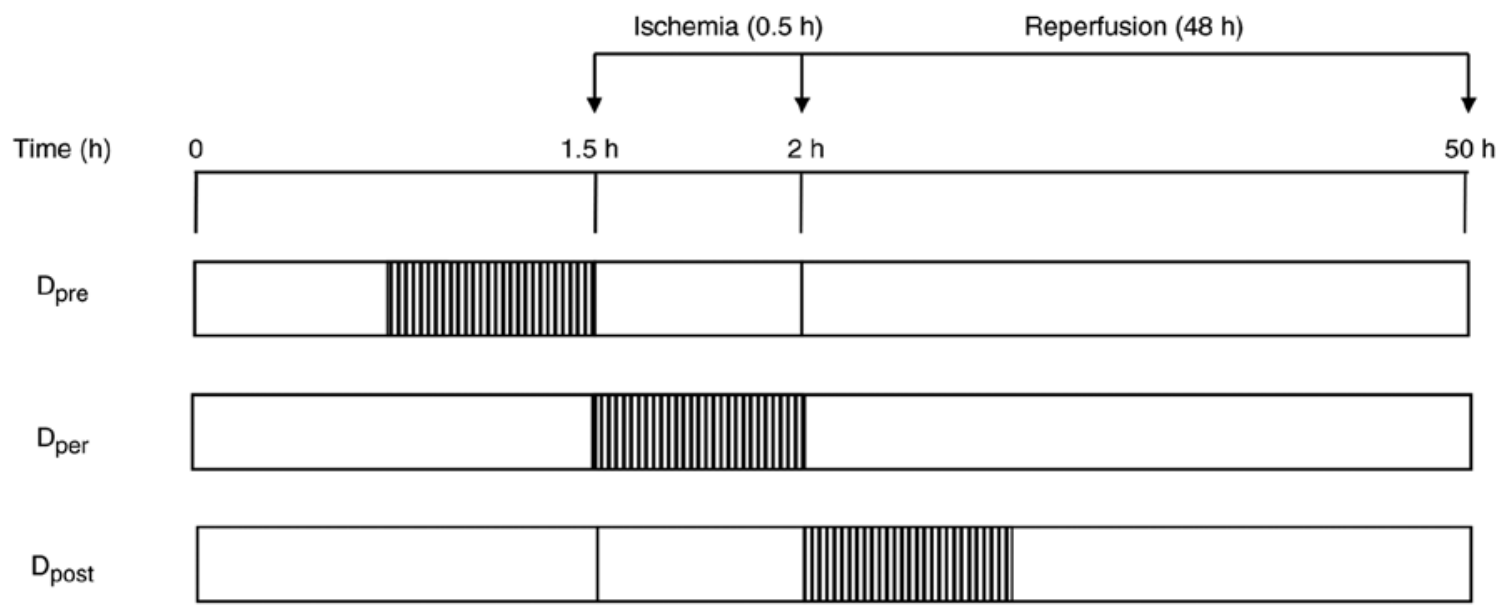

Drug infusion for $0.5 \mathrm{~h}$

Figure 1. Diagrammatic presentation of the experimental protocol. $\mathrm{D}_{\text {pre }}$, DADLE pre-conditioning; $\mathrm{D}_{\text {post }}$, DADLE post-conditioning; $\mathrm{D}_{\text {per, }}$, DADLE per-conditioning; DADLE, D-Ala ${ }^{2}$, D-Leu ${ }^{5}$-Enkephalin.

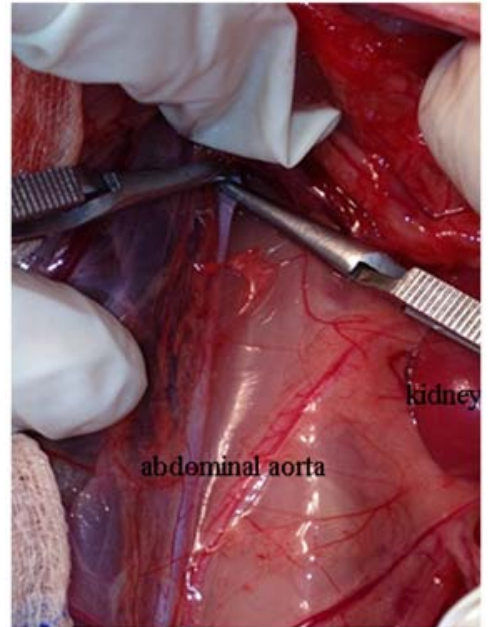

Figure 2. Abdominal aorta occlusion with artery clamps. The infrarenal abdominal aorta was exposed via abdominal incision with the ligatures placed loosely around it. A polycarbonate catheter (20-G) was inserted into the aorta via femoral artery incision with the tip reaching $1-2 \mathrm{~cm}$ below the left renal artery. To achieve spinal cord ischemia, the infrarenal abdominal aorta was blocked with two artery clips.

type I error rate for multiple comparisons. The incidences of paraplegia and neurological dysfunction were compared using a Fisher's exact test followed by Bonferroni correction. The Bonferroni-adjusted P-value was obtained by multiplying the unadjusted P-value by the comparisons number (i.e., 3), and was presented as the 'corrected P'. Corrected $\mathrm{P}<0.05$ was considered to indicate a statistically significant result. The number of viable neurons is expressed as the median (25 and 75 th percentiles). Tarlov scores are presented in absolute numbers. All statistical tests were two-tailed.

\section{Results}

DADLE per-conditioning attenuates spinal cord I/R injury. Tarlov scores of the Sham, NS and $D_{\text {per }}$ groups at different time points following reperfusion are presented in Fig. 3. All rabbits in the Sham group retained unimpaired neurological functions; however, spinal cord I/R injury induced significant neurological dysfunction [corrected $\mathrm{P}=0.0006$ at all different time points following reperfusion (palinesthesia, and 6, 24 and 48 h); Fig. 3]. Compared with the NS group, the animals that received DADLE perfusion demonstrated significantly improved neurological deficits (corrected $\mathrm{P}=0.0006$ at the time points of palinesthesia; $\mathrm{P}=0.0009$ at $6 \mathrm{~h} ; \mathrm{P}=0.0141$ at $24 \mathrm{~h}$; and $\mathrm{P}=0.0270$ at $48 \mathrm{~h}$ after reperfusion). The paraplegia rates were significantly decreased from $87.5 \%$ in the NS group to $25 \%$ in the DADLE per-conditioning group at $48 \mathrm{~h}$ after the reperfusion ( $\mathrm{P}=0.0400$; data not shown).

Representative images of sections stained with hematoxylin and eosin of the Sham, NS and $\mathrm{D}_{\text {per }}$ groups are presented in Fig. 4A-C. According to the cell count, I/R injuries demonstrated a significant increase in the amount of damaged neurons compared with the Sham group (corrected $\mathrm{P}=0.0006$; Fig. 4D). The median number of viable $\alpha$-motor neurons at $48 \mathrm{~h}$ after reperfusion was 3.5 (range, 2-4) in the $\mathrm{D}_{\text {per }}$ group, which was significantly higher than the median value of 0.5 (range, 0-1) in the NS group (corrected $\mathrm{P}=0.0060$ ), but lower than the median value of 7 (range, 5.25-7) in the Sham group (corrected $\mathrm{P}=0.0018$ ). The results of the Tarlov scores and histopathological examination of the spinal cords of the rabbits that received DADLE were consistent with a previous study (15).

Effects of DADLE pre-and post-conditioning on spinal cord I/R injury

Physiologic parameters. The average weight and the core temperature of the animals were not significantly different between the three groups, as presented in Table I. HR and MAP were observed and maintained in each group, as presented in Table II.

Neurological outcomes. The Tarlov scores of Group $\mathrm{D}_{\mathrm{per}}$, Group $\mathrm{D}_{\text {pre }}$ and Group $\mathrm{D}_{\text {post }}$ are presented in Fig. 5. DADLE 
Palinesthesia

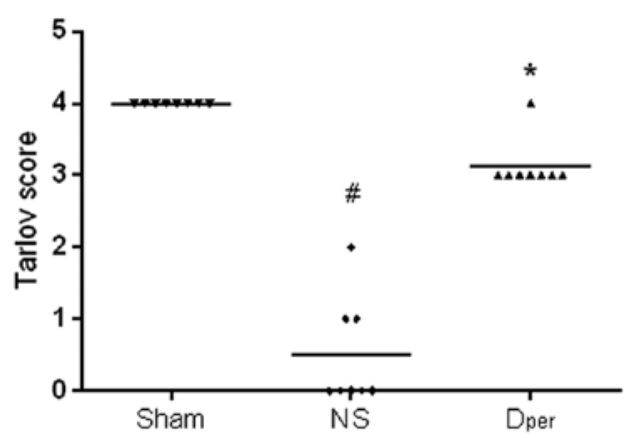

$24 \mathrm{~h}$ after reperfusion

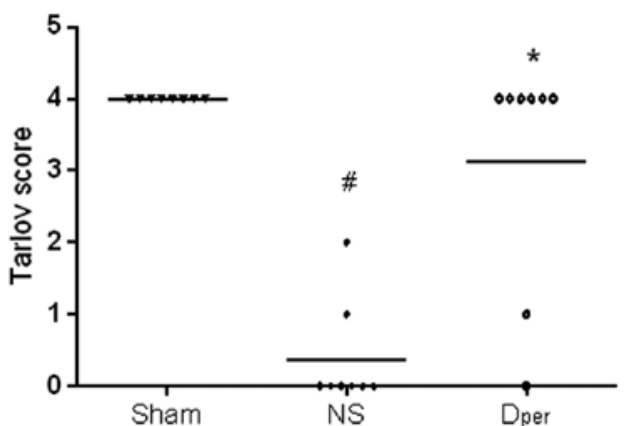

$6 \mathrm{~h}$ after reperfusion

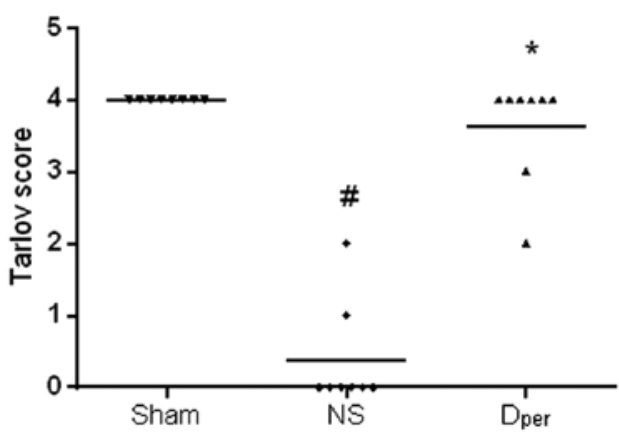

$48 \mathrm{~h}$ after reperfusion

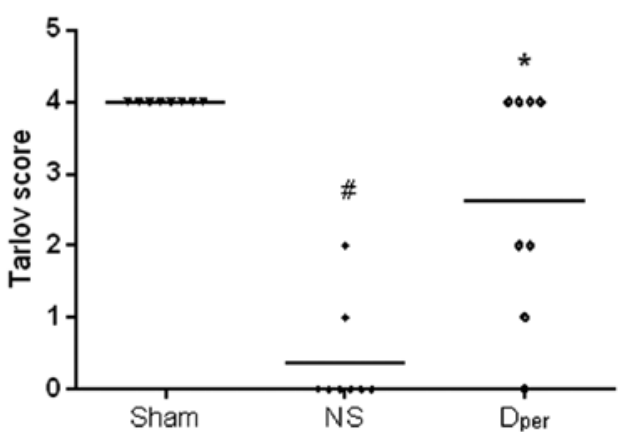

Figure 3. Tarlov scores of the Sham, NS and $\mathrm{D}_{\text {per }}$ groups at different time points following reperfusion. Spinal cord I/R injury induced significant neurological dysfunction. DADLE per-conditioning improved neurological outcome following spinal cord I/R injury at different time points following reperfusion. ${ }^{*}$ Corrected $\mathrm{P}=0.0006$ at the time points of palinesthesia; $\mathrm{P}=0.0009$ at $6 \mathrm{~h} ; \mathrm{P}=0.0141$ at $24 \mathrm{~h}$; and $\mathrm{P}=0.0270$ at $48 \mathrm{~h}$ following reperfusion vs. NS. ${ }^{\#}$ Corrected $\mathrm{P}=0.0006$ vs. Sham group for all different time points (palinesthesia, and 6, 24 and 48 h). Bars represent the mean. Sham, Sham-operated; NS, normal saline; $\mathrm{D}_{\text {per }}$, DADLE per-conditioning; DADLE, D-Ala ${ }^{2}$, D-Leu ${ }^{5}$-Enkephalin; I/R, ischemia and reperfusion.

A

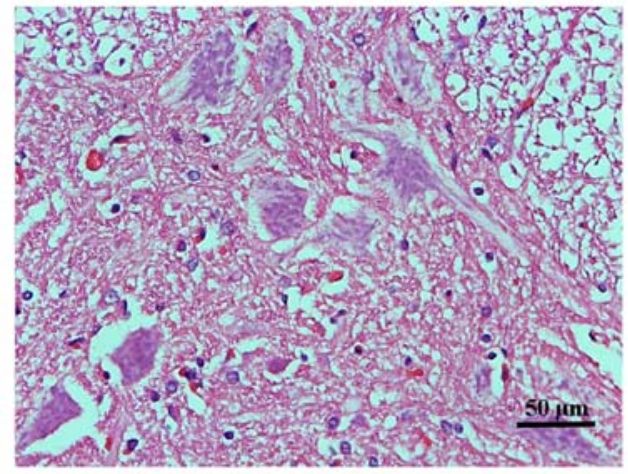

C

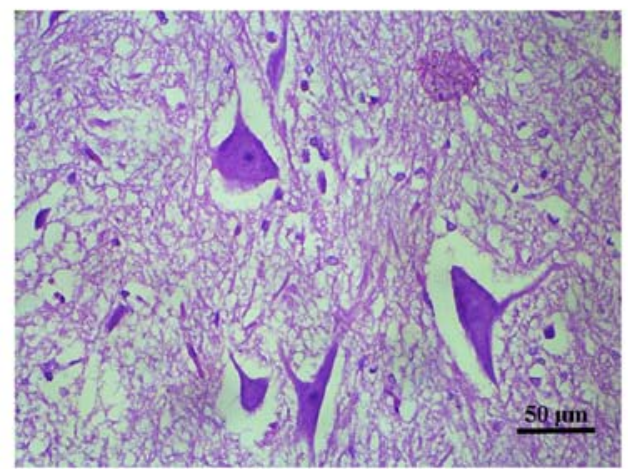

B

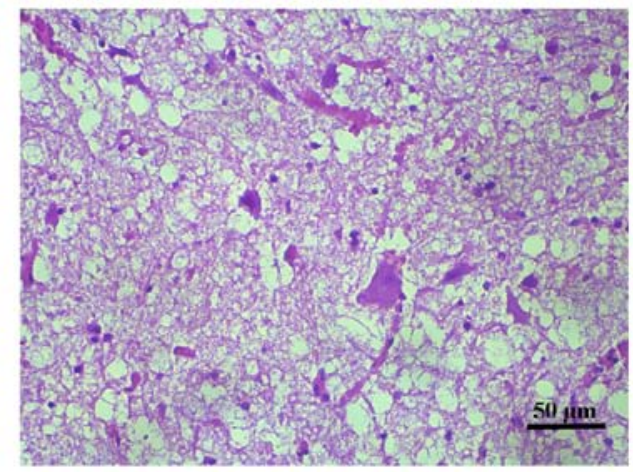

D

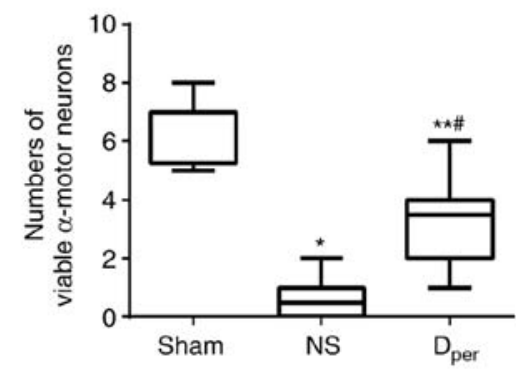

Figure 4. Hematoxylin and eosin staining in the anterior horn of spinal cord of the Sham, NS and $\mathrm{D}_{\text {per }}$ groups at $48 \mathrm{~h}$ after reperfusion. (A) Sham group exhibited an abundance of normal neurons, while (B) the NS group demonstrated extensive necrosis and decreased viable neurons. (C) Normal neuronal injuries were mitigated in the $\mathrm{D}_{\text {per }}$ group (magnification, $\mathrm{x} 40$ ). (D) Numbers of viable $\alpha$-motor neurons in the anterior horn of spinal cord. *Corrected $\mathrm{P}=0.0006$, ${ }^{* *}$ corrected $\mathrm{P}=0.0018$ vs. Sham; ${ }^{*}$ corrected $\mathrm{P}=0.0060$ vs. NS. Bars represent the interquartile range. Sham, Sham-operated; NS, normal saline; $\mathrm{D}_{\text {per }}$, DADLE per-conditioning; DADLE, D-Ala ${ }^{2}$, D-Leu ${ }^{5}$-Enkephalin. 
Table I. Body weight and core temperature of the rabbits.

Core temperature, ${ }^{\circ} \mathrm{C}$

Group ( $\mathrm{n}=8$ per group) Body weight, g P-value Pre-ischemia P-value Intra-ischemia P-value Post-ischemia P-value

\begin{tabular}{|c|c|c|c|c|c|c|c|c|}
\hline $\mathrm{D}_{\text {per }}$ & $2612 \pm 260$ & & $37.3 \pm 0.8$ & & $37.1 \pm 0.9$ & & $37.2 \pm 0.8$ & \\
\hline $\mathrm{D}_{\text {pre }}$ & $2670 \pm 280$ & 0.83 & $37.4 \pm 0.7$ & 0.95 & $37.3 \pm 0.9$ & 0.90 & $37.3 \pm 0.7$ & 0.95 \\
\hline$D_{\text {post }}$ & $2590 \pm 265$ & & $37.3 \pm 0.6$ & & $37.2 \pm 0.8$ & & $37.2 \pm 0.6$ & \\
\hline
\end{tabular}

Data values are expressed as the mean \pm standard deviation. There were no significant differences in the body weight and core temperature between the groups $(\mathrm{P}>0.05)$. $\mathrm{D}_{\text {per }}$, DADLE per-conditioning; $\mathrm{D}_{\text {pre }}$, DADLE pre-conditioning; $\mathrm{D}_{\text {post }}$, DADLE post-conditioning; DADLE, D-Ala ${ }^{2}$, D-Leu ${ }^{5}$-Enkephalin.

per-conditioning improved neurological outcome at different time points following reperfusion. The Tarlov scores of DADLE perfusion were then compared during ischemia, prior to ischemia onset or at the time of early reperfusion in order to determine the optimum time point of administration. The results revealed that there were no significant differences between the three groups ( $\mathrm{P}>0.05$; Fig. 5). However, the Tarlov scores were higher in the DADLE per- and post-conditioning groups compared with those in the DADLE pre-conditioning group, but these were not statistically significant (corrected $\mathrm{P}>0.05$; Fig. 5). The paraplegia rates are summarized in Table III. The rates of paraplegia and neurological dysfunction were determined as above. In the $\mathrm{D}_{\text {per }}$ group and the $\mathrm{D}_{\text {post }}$ group, $25 \%$ rabbits suffered from hind-limb paraplegia at $48 \mathrm{~h}$ after the reperfusion compared with $62.5 \%$ rabbits in the $\mathrm{D}_{\text {pre }}$ group $(\mathrm{P}>0.05)$. In addition, the results revealed that there were no significant differences in the incidences of neurological dysfunction between the three groups. However, the neurological dysfunction rates were higher in the $\mathrm{D}_{\text {pre }}$ group when compared with the $\mathrm{D}_{\text {per }}$ and $\mathrm{D}_{\text {post }}$ groups at 6,24 and $48 \mathrm{~h}$ after the reperfusion, but these were also not statistically significant ( $\mathrm{P}>0.05$; Table III).

Histopathologicalchanges in the anterior horn. Representative images of sections stained with hematoxylin and eosin are presented in Fig. 6A-C. There was a greater number of viable $\alpha$-motor neurons observed in the $D_{\text {per }}$ and $D_{\text {post }}$ group than the $\mathrm{D}_{\text {pre }}$ group, but this was not statistically significant ( $P>0.05$; Fig. 6D). The number of normal $\alpha$-motor neurons appeared largest in the post-conditioning group with DADLE, and least in the pre-conditioning group. These histopathological changes were closely associated with the neurological outcomes, suggesting that DADLE pre- and post-conditioning offer a method of preservation of normal neurons that is just as effective as per-conditioning.

\section{Discussion}

The rabbit model utilized in the present study was established based on a classical model and previous research $(20,21)$. It has been demonstrated that spinal cord injury is relatively consistent with infrarenal aortic occlusion for $30 \mathrm{~min}$ and the paraplegia rate can approach $\sim 80 \%$. In contrast to systematic administration, intra-aortic administration presents clear advantages, such as improved protective efficiency of the spinal cord and low systemic side effects. The biggest advantage of this method is that a much higher pharmaceutical concentration of DADLE can be infused directly to the ischemic spinal cord segments through the lumbar arteries (22).

A previous study demonstrated that regional administration of DADLE via the abdominal aorta provided dose-dependent protection on spinal cord I/R in rabbits (15). After studying the dose-dependent protective effect, it was revealed that the time of drug administration was also acritical factor for a neuroprotective effect. Considering the advantages of pharmacological pre- and post-conditioning in the prevention and treatment of patients with ischemic events in the clinical setting, a new experiment was designed. The purposes of the previous and the present study are different. In the present study, the neuroprotective effects of DADLE administration were compared at different time periods, i.e., before, during and after ischemia in order to determine which conditioning strategy was the best.

Similar to previous findings, the results from the present study revealed that DADLE per-conditioning provided greater protection than NS. In addition, it was demonstrated that DADLE perfusion at the other two time points also provided neuroprotection of the brain against I/R injury. However, the Tarlov scores and the incidences of paraplegia in the rabbits treated with DADLE per- and post-conditioning were higher than those subjected to DADLE pre-conditioning, despite not being statistically significant. DADLE post-conditioning markedly decreased normal motor neuron injury and improved the neurologic deficit scores $48 \mathrm{~h}$ after spinal cord ischemia in the present study. Such effective protection of DADLE post-conditioning in the spinal cord was in accordance with that reported in the brain (14) and heart (23). The results from the present study suggested that DADLE can be applied following ischemia occurrence, which can not only protect the spinal cord from ischemia, but is also more practical in the clinical setting compared with per-conditioning, and provides a better therapeutic option.

Ischemic pre- and post-conditioning have emerged as useful new strategies for ameliorating organ injuries, preserving associated functions and potentially improving morbidity and mortality (24). Ischemic pre-conditioning, as the first-used form of pre-conditioning, is an adaptive response triggered 

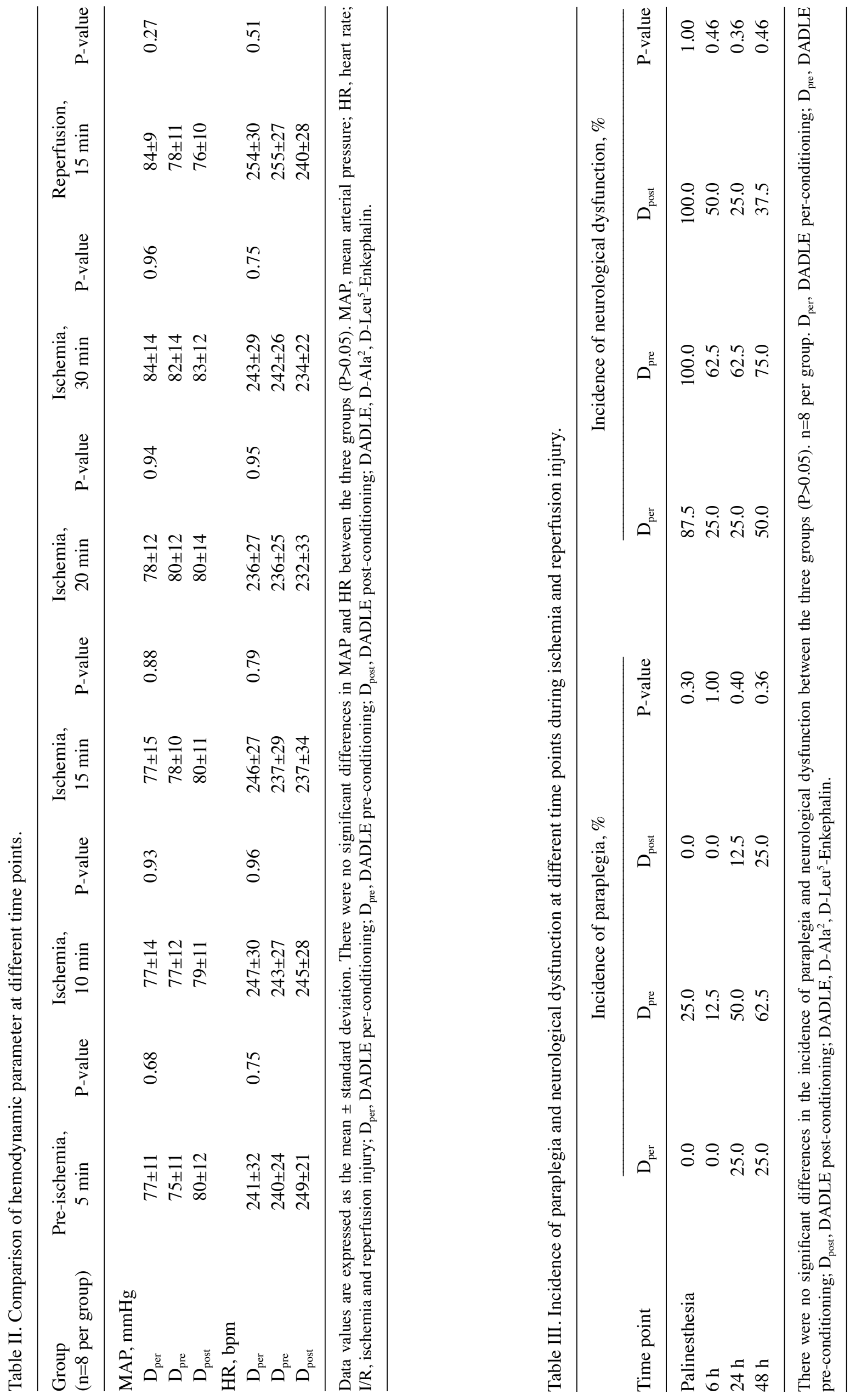

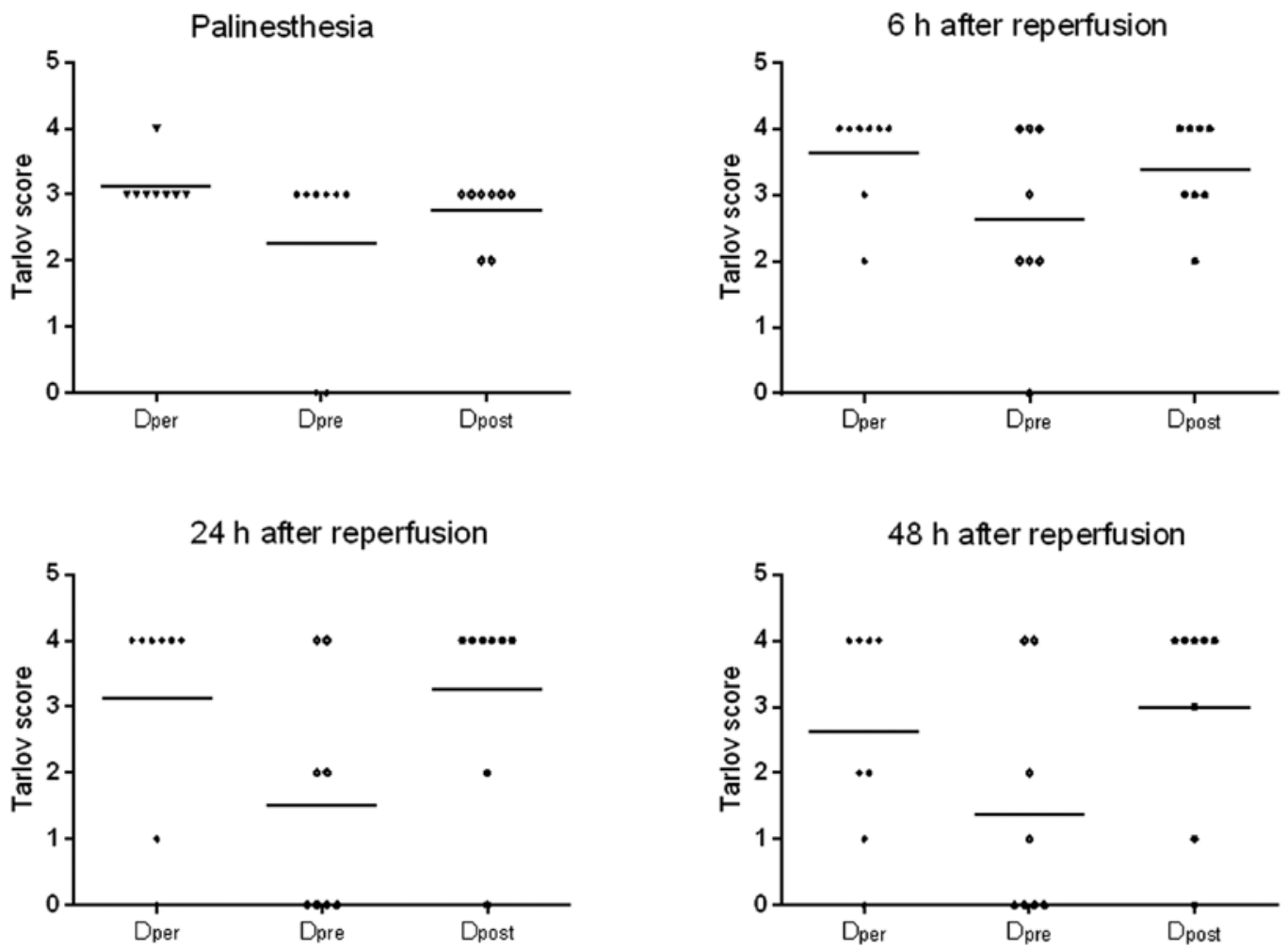

Figure 5. Tarlov scores of the DADLE per-, pre- and post-conditioning groups at different time points following reperfusion. DADLE per-, pre-and post-conditioning improved neurological outcome following spinal cord ischemia and reperfusion injury at different time points after reperfusion. There was no significant difference in Tarlov scores among groups. Bars represent the mean. $\mathrm{D}_{\text {pre }}$, DADLE pre-conditioning; $\mathrm{D}_{\text {post }}$, DADLE post-conditioning; $\mathrm{D}_{\text {per }}$, DADLE per-conditioning; DADLE, D-Ala ${ }^{2}$, D-Leu ${ }^{5}$-Enkephalin.

A

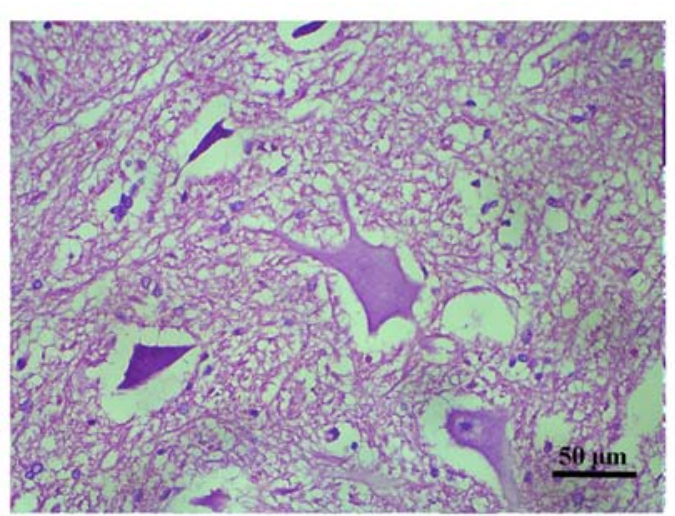

C

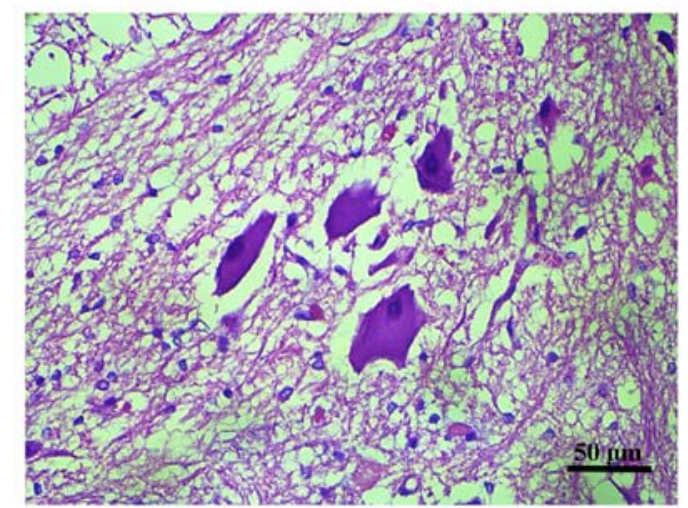

B

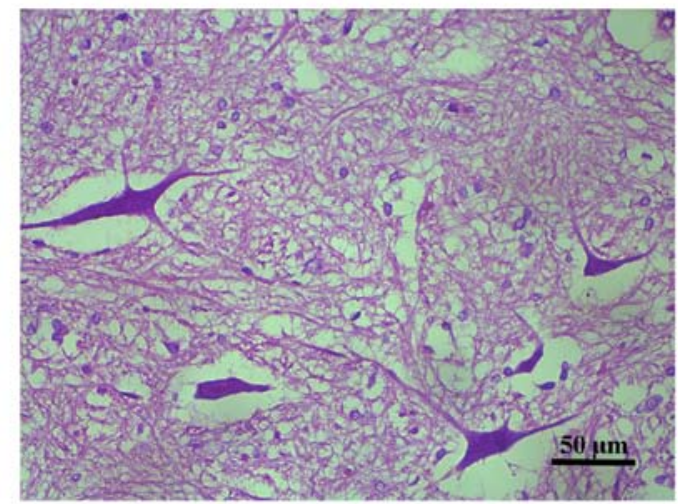

D

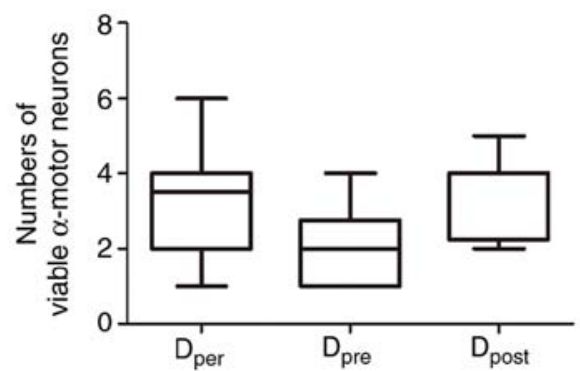

Figure 6. Hematoxylin and eosin staining in the anterior horn of spinal cord of the DADLE per-, pre-and post-conditioning groups at $48 \mathrm{~h}$ after reperfusion. There was a greater number of normal neurons observed in (A) the $\mathrm{D}_{\text {per }}$ and (C) $\mathrm{D}_{\text {post }}$ groups compared with the (B) $\mathrm{D}_{\text {pre }}$ group (magnification, $\mathrm{x} 40$ ). (D) Numbers of viable $\alpha$-motor neurons in the anterior horn of spinal cord. There was no significant difference in numbers of viable $\alpha$-motor neurons between the three groups. Bars represent the results of interquartile range. $\mathrm{D}_{\mathrm{pre}}$, DADLE pre-conditioning; $\mathrm{D}_{\text {post }}$, DADLE post-conditioning; $\mathrm{D}_{\text {per }}$, DADLE per-conditioning; DADLE, D-Ala² ${ }^{2}$ D-Leu ${ }^{5}$-Enkephalin. 
by brief ischemia applied prior to prolonged ischemia, and has been demonstrated to have a powerful protective effect against I/R injury for the spinal cord $(25,26)$. Later, ischemic post-conditioning via mechanical interruptions of reperfusion reported by Zhao et al (27) was as effective as ischemic pre-conditioning in decreasing infarct size in open-chest dogs. However, these mechanical approaches are invasive in nature, and there are inherent risks of thromboembolism and damage with repeated clamping and declamping of the aorta. Therefore, pharmacological methods became the focus of the experiments to assess the anticipated I/R injury of an organ or tissue. In several previous studies, a variety of diverse pharmacological pre- and post-conditioning agents, such as adenosine, natriuretic peptide and bradykinin led to beneficial organ protective effects (4-6). Among these agents, opioid pre-and post-conditioning as opposed to transient ischemic stimuli have now been demonstrated to elicit a satisfactory protective effect against ischemia from the heart to the brain in animal models $(9,11,28)$.

DADLE, an artificial synthetic delta opioid receptor agonist, has been demonstrated to prevent the central nervous system from ischemic injury in cultured cells and animal experiments $(13,29)$. For example, Su et al (13) demonstrated that intracerebroventricular administration of DADLE $45 \mathrm{~min}$ before forebrain ischemia had a protective effect against hippocampal CA1 neurons loses and a dose-dependent improvement of intellectual disability. In addition, the authors revealed that DADLE administered at the onset of reperfusion also exhibited a preservation of CA1 neurons and cognitive benefits in rats with transient forebrain ischemia (14). However, to the best of our knowledge, there are no studies currently published that investigate the effects on the spinal cord. Therefore, the present study was designed and a positive result was observed. From the perspective of clinical application, the utilization of drug post-conditioning is more practical considering the unpredictability of disease occurrence. Post-conditioning with DADLE may be more feasible when applying the technique to post-ischemic spinal cord tissue.

In the present study, the paraplegia rate and loss of normal motor neurons were higher in the DADLE pre-conditioning compared with the DADLE per- and post-conditioning groups, although these results were not statistically significant. Previously, Su et al (13) demonstrated that DADLE provided protection when administered $45 \mathrm{~min}$ before ischemia, which was different to what was observed in the present study. These differences may be due to the different animal models used. Lee and Amidon (30) demonstrated that the half-lives of DADLE in distribution and elimination phases were very short, $\sim 0.5$ and $5 \mathrm{~min}$, respectively. DADLE reached plateau plasma concentration within 15 min of intravenous administration and was rapidly cleared following absorption. Therefore, mitigated neuroprotection of DADLE pre-conditioning might be due to its short half-life.

DADLE treatment is known to result in transient depression of MAP and HR in spinal cord I/R injury (17). In this study, it was revealed that the MAP was lower at the point of pre-ischemia compared with the point of ischemia and reperfusion in the DADLE pre-conditioning group; however, this was not statistically significant. Similarly, it was also observed that MAP and HR were lower at the point of post-ischemia compared with the point of pre-ischemia and during ischemia in the DADLE post-conditioning group; however, again, this was not statistically significant. The statistical significances of HR among the three groups were not determined. Two reasons may account for this phenomenon. Firstly, a relatively low-dose of DADLE may lead to fewer hemodynamic changes took place in rabbits. Secondly, NS was infused intravenously at a rate of $10 \mathrm{ml} / \mathrm{kg}$ per $\mathrm{h}$ to maintain fluid requirements in the present study.

Furthermore, whether the results of the present study could be applied to a clinical setting remains unknown. American Spinal Injury Association (ASIA) guidelines (31) are used for the assessment of patient motor, sensory and autonomic dysfunction following spinal cord injury that should be assessed by clinicians. The present study used the Tarlov scoring system instead of ASIA for neurological evaluation, as the source of blood supply to the spinal cord of rabbits is different from that in humans. The homosegmental blood supply of spinal cords in rabbits begins at the abdominal aorta caudally to the origin of renal arteries with minimal or no intraspinal collateral arterial system. However, the blood supply to the spinal cord in humans originates from segmental arteries (lumbar arteries) and the vertebral artery (32). The Tarlov score is a widely accepted and matched method for evaluation of rabbit neural dysfunction following spinal cord injury (33). A significant benefit was observed when DADLE was administered after the start of abdominal aorta occlusion, even at an early phase of reperfusion in rabbits. The results from the present study may provide an innovative therapeutic strategy for a clinical situation.

There are several limitations to the present study. First, the present study only investigated the effectiveness of the strategies in question and, therefore, the underlying molecular mechanisms remain unclear. Tian et al (34) reported that DADLE was able to inhibit cellular transcription by regulating phosphorylation of RNA polymerase II in primary cortical neurons, which may provide a potential insight into the molecular mechanism underlying neuroprotection. In the present study, it was revealed that the protective effects of DADLE per-conditioning may be associated with its anti-oxidant and anti-apoptotic properties in the rabbit model of spinal cord I/R injury (35). Future studies may aim to clarify the potential mechanisms responsible for the different protective effects of DADLE pre- and post-conditioning.

In summary, the present study suggested that DADLE administration at three time points, before ischemia onset, during the ischemic period or at the early reperfusion period for $30 \mathrm{~min}$ exerted preservation effects on neurological function and normal neurons in a rabbit model of spinal cord I/R. The therapeutic effects appeared most notable in the post-conditioning group with DADLE, and was worst in the pre-conditioning group. The results from the present study may provide new therapeutic potentials in improving clinical outcomes in patients with thoraco-abdominal aortic cross-clamping.

\section{Acknowledgements}

Not applicable. 


\section{Funding}

The present study was funded by The National Natural Science Foundation of China (grant. no. 81771269), The Shanghai Pujiang Program (grant. no. 17PJD035) and The Shanghai Natural Science Foundation (grant no. 15ZR1433800).

\section{Availability of data and materials}

The datasets used and/or analyzed during the current study are available from the corresponding author on reasonable request.

\section{Authors' contributions}

JY and HuL conceived and designed the experiments. DF performed the experiments, analyzed the data and wrote the manuscript. HaL performed the experiments and contributed to the reagents, materials and analysis tools.

\section{Ethics approval and consent to participate}

The animal protocol was approved by The Animal Care and Use Committee of Shanghai Jiaotong University.

\section{Patient consent for publication}

Not applicable.

\section{Competing interests}

The authors declare that they have no competing interests.

\section{References}

1. Zvara DA: Thoracoabdominal aneurysm surgery and the risk of paraplegia: Contemporary practice and future directions. J Extra Corpor Technol 34: 11-17, 2002.

2. Ballard JL: Thoracoabdominal aortic aneurysm repair: Historical review and description of a re-engineered technique. Perspect Vasc Surg Endovasc Ther 17: 207-215, 2005.

3. Mehmedagic I, Resch T and Acosta S: Complications to cerebrospinal fluid drainage and predictors of spinal cord ischemia in patients with aortic disease undergoing advanced endovascular therapy. Vasc Endovascular Surg 47: 415-422, 2013.

4. Kitakaze M, Asakura M, Kim J, Shintani Y, Asanuma H, Hamasaki T, Seguchi O, Myoishi M, Minamino T, Ohara T, et al Human atrial natriuretic peptide and nicorandil as adjuncts to reperfusion treatment for acute myocardial infarction (J-WIND) Two randomised trials. Lancet 370: 1483-1493, 2007.

5. Kloner RA, Forman MB, Gibbons RJ, Ross AM, Alexander RW and Stone GW: Impact of time to therapy and reperfusion modality on the efficacy of adenosine in acute myocardial infarction: The AMISTAD-2 trial. Eur Heart J 27: 2400-2405, 2006.

6. Danielisova V, Gottlieb M, Nemethova M, Kravcuková P, Domoráková I, Mechírová E and Burda J: Bradykinin postconditioning protects pyramidal CA1 neurons against delayed neuronal death in rat hippocampus. Cell Mol Neurobiol 29: 871-878, 2009.

7. Li L and Zuo Z: Isoflurane postconditioning induces neuroprotection via Akt activation and attenuation of increased mitochondrial membrane permeability. Neuroscience 199: 44-50, 2011.

8. Yu QJ, Zhou QS, Huang HB, Wang YL, Tian SF and Duan DM: Protective effect of ketamine on ischemic spinal cord injury in rabbits. Ann Vasc Surg 22: 432-439, 2008.

9. Charron C, Messier C and Plamondon H: Neuroprotection and functional recovery conferred by administration of kappa- and delta 1-opioid agonists in a rat model of global ischemia. Physiol Behav 93: 502-511, 2008
10. Schultz JE, Rose E, Yao Z and Gross GJ: Evidence for involvement of opioid receptors in ischemic preconditioning in rat hearts. Am J Physiol 268: H2157-H2161, 1995.

11. Zatta AJ, Kin H, Yoshishige D, Jiang R, Wang N, Reeves JG, Mykytenko J, Guyton RA, Zhao ZQ, Caffrey JL and Vinten-Johansen J: Evidence that cardioprotection by postconditioning involves preservation of myocardial opioid content and selective opioid receptor activation. Am J Physiol Heart Circ Physiol 294: H1444-H1451, 2008.

12. Borlongan CV, Wang Y and Su TP: Delta opioid peptide (D-Ala 2, D-Leu 5) enkephalin: Linking hibernation and neuroprotection. Front Biosci 9: 3392-3398, 2004.

13. Su DS, Wang ZH, Zheng YJ, Zhao YH and Wang XR: Dose-dependent neuroprotection of delta opioid peptide [D-Ala2, D-Leu5] enkephalin in neuronal death and retarded behavior induced by forebrain ischemia in rats. Neurosci Lett 423: 113-117, 2007.

14. Wang S, Duan Y, Su D, Li W, Tan J, Yang D, Wang W, Zhao Z and Wang X: Delta opioid peptide [D-Ala2, D-Leu5] enkephalin (DADLE) triggers postconditioning against transient forebrain ischemia. Eur J Pharmacol 658: 140-144, 2011.

15. Liu H, Chen B, Li S and Yao J: Dose-dependent neuroprotection of delta-opioid peptide [D-Ala(2), D-Leu(5)] enkephalin on spinal cord ischemia-reperfusion injury by regional perfusion into the abdominal aorta in rabbits. J Vasc Surg 63: 1074-1081, 2016.

16. National Research Council (US) Institute for Laboratory Animal Research: Guide for the care and use of laboratory animals. Washington (DC), National Academies Press (US), 1996.

17. Liu H, Chen B, Zhang Y, Qiu Y, Xia Y, Li S and Yao J: Protective effect of delta opioid agonist [D-Ala2, D-Leu5] enkephalin on spinal cord ischemia reperfusion injury by regional perfusion into abdominal aorta in rabbits. Neurosci Lett 584: 1-6, 2015.

18. Tarlov IM: Acute spinal cord compression paralysis. J Neurosurg 36: 10-20, 1972.

19. Ehrlich M,Knolle E, Ciovica R, Böck P, Turkof E, Grabenwöger M, Cartes-Zumelzu F, Kocher A, Pockberger H, Fang WC, et al: Memantine for prevention of spinal cord injury in a rabbit model. J Thorac Cardiovasc Surg 117: 285-291, 1999.

20. Apaydin AZ and Buket S: Regional lidocaine infusion reduces postischemic spinal cord injury in rabbits. Tex Heart Inst J 28: 172-176, 2001.

21. Yao JY, Weng H, Zhang L, Wang QY, Yuan YQ, Tang Y and Li JS: The reperfusion injury model improvement and the tolerance time investigation of rabbit spinal cord ischemia under normothermia. Sichuan Da Xue Xue Bao Yi Xue Ban 38: 497-500, 542, 2007 (In Chinese)

22. Hamaishi M, Orihashi K, Isaka M, Kumagai H, Takahashi S, Okada K, Ohtaki M and Sueda T: Low-dose edaravone injection into the clamped aorta prevents ischemic spinal cord injury. Ann Vasc Surg 23: 128-135, 2009.

23. Fuardo M, Lemoine S, Lo Coco C, Hanouz JL and Massetti M [D-Ala2,D-Leu5]-enkephalin (DADLE) and morphine-induced postconditioning by inhibition of mitochondrial permeability transition pore, in human myocardium. Exp Biol Med (Maywood) 238: 426-432, 2013.

24. Hausenloy DJ and Yellon DM: Preconditioning and postconditioning: Underlying mechanisms and clinical application. Atherosclerosis 204: 334-341, 2009.

25. Toumpoulis IK, Papakostas JC,Matsagas MI, Malamou-Mitsi VD, Pappa LS, Drossos GE, Derose JJ and Anagnostopoulos CE: Superiority of early relative to late ischemic preconditioning in spinal cord protection after descending thoracic aortic occlusion. J Thorac Cardiovasc Surg 128: 724-730, 2004.

26. Zvara DA, Colonna DM, Deal DD, Vernon JC, Gowda M and Lundell JC: Ischemic preconditioning reduces neurologic injury in a rat model of spinal cord ischemia. Ann Thorac Surg 68: 874-880, 1999

27. Zhao ZQ, Corvera JS, Halkos ME, Kerendi F, Wang NP, Guyton RA and Vinten-Johansen J: Inhibition of myocardial injury by ischemic postconditioning during reperfusion: Comparison with ischemic preconditioning. Am J Physiol Heart Circ Physiol 285: H579-H588, 2003.

28. Chen Z, Li T and Zhang B: Morphine postconditioning protects against reperfusion injury in the isolated rat hearts. J Surg Res 145: 287-294, 2008.

29. Ma MC, Qian H, Ghassemi F, Zhao P and Xia Y: Oxygen-sensitive \{delta\}-opioid receptor-regulated survival and death signals: Novel insights into neuronal preconditioning and protection. J Biol Chem 280: 16208-16218, 2005. 
30. Lee HJ and Amidon GL: The effect of enzyme inhibitor and absorption site following [D-ala2, D-leu5]enkephalin oral administration in rats. Biopharm Drug Dispos 23: 131-141, 2002.

31. Krassioukov AV, Karlsson AK, Wecht JM, Wuermser LA, Mathias CJ and Marino RJ; Joint Committee of American Spinal Injury Association and International Spinal Cord Society: Assessment of autonomic dysfunction following spinal cord injury: Rationale for additions to international standards for neurological assessment. J Rehabil Res Dev 44: 103-112, 2007.

32. Santillan A, Nacarino V, Greenberg E, Riina HA, Gobin YP and Patsalides A: Vascular anatomy of the spinal cord. J Neurointerv Surg 4: 67-74, 2012.

33. Seppälä M, Antinheimo J, Pohjola J and Hernesniemi J: Acute spinal cord compression. Duodecim 129: 2655-2660, 2013 (In Finnish).
34. Tian J, Gu Y, Sun K, Wang B, Chen J, Wang X and Su D: [D-Ala2, D-Leu5] encephalin (DADLE) reversibly inhibits cellular transcription in neurons without causing cell injury. Brain Res 1565: $1-7,2014$.

35. Fu D, Liu H, Li S, Chen L and Yao J: Antioxidative and antiapoptotic effects of delta-opioid peptide [D-Ala $\left.{ }^{2}, \mathrm{D}-\mathrm{Leu}^{5}\right]$ enkephalin on spinal cord ischemia-reperfusion injury in rabbits. Front Neurosci 11: 603, 2017.

This work is licensed under a Creative Commons Attribution-NonCommercial-NoDerivatives 4.0 International (CC BY-NC-ND 4.0) License. 